\title{
THE NEWTON POLYTOPE OF THE IMPLICIT EQUATION
}

\author{
BERND STURMFELS, JENIA TEVELEV, AND JOSEPHINE YU \\ Dedicated to Askold Khovanskii on the occasion of his 60th birthday
}

\begin{abstract}
We apply tropical geometry to study the image of a map defined by Laurent polynomials with generic coefficients. If this image is a hypersurface then our approach gives a construction of its Newton polytope.
\end{abstract}

\section{INTRODUCTION}

Implicitization is a fundamental operation in computational algebraic geometry. Its objective is to transform a given parametric representation of an algebraic variety into its implicit representation as the zero set of polynomials. Most algorithms for implicitization are based on multivariate resultants or Gröbner bases, but current implementations of these algorithms tend to be too slow to handle large instances.

Adopting Khovanskii's philosophy on the importance of Newton polytopes [Kho, the foremost case of the implicitization problem is as follows. Let $f_{1}, \ldots, f_{n} \in$ $\mathbb{C}\left[t_{1}^{ \pm 1}, \ldots, t_{d}^{ \pm 1}\right]$ be Laurent polynomials with Newton polytopes $P_{1}, \ldots, P_{n} \subset \mathbb{R}^{d}$ and with supports $A_{i} \subset P_{i}$. We assume that each $f_{i}$ is generic relative to its support, which means that the coefficient vector of $f_{i}$ lies in a Zariski open subset of $\mathbb{C}^{A_{i}}$. The aim of implicitization is to compute the prime ideal $I$ of all polynomials $g \in \mathbb{C}\left[x_{1}, \ldots, x_{n}\right]$ which satisfies $g\left(f_{1}, \ldots, f_{n}\right) \equiv 0$ in $\mathbb{C}\left[t_{1}^{ \pm 1}, \ldots, t_{d}^{ \pm 1}\right]$. We here seek to read off as much information as possible about the variety $V(I)$ from $P_{1}, \ldots, P_{n}$.

Of particular interest is the case $n=d+1$, when $I=\langle g\rangle$ is a principal ideal. Here the problem is to predict the Newton polytope $Q$ of the hypersurface $V(I)=$ $\{g=0\}$ from $P_{1}, \ldots, P_{n}$. This problem was posed in $[\mathrm{SY}]$ and has remained open for over a decade. It also reappeared in recent work of Emiris and Kotsireas EK.

We here present a general solution to the problem of [SY], namely, a construction of the Newton polytope $Q$ in terms of the input polytopes $P_{i}$. When the variety $V(I)$ is not a hypersurface but has codimension greater than one, the role of $Q$ is played by the Chow polytope KSZ, and our construction generalizes to that case.

The present exposition is not self-contained; it refers for two proofs to forthcoming papers. Our calculations are based on geometric characterizations of $\mathcal{T}(I)$ derived by Hacking, Keel and Tevelev in HKT Te. A theory of Tropical Implicitization, which further develops these results, appears in STY. In that theory, the coefficients of the $f_{i}(t)$ can be arbitrary (non-generic) complex numbers, or even scalars in an arbitrary field with a non-archimedean valuation. One objective of this paper is to announce and explain tropical implicitization to a wider audience.

2000 Mathematics Subject Classification. 13P10, 14Q99, 52B20, 68W30.

Key words and phrases. Implicitization, Newton polytope, Tropical geometry.

Josephine Yu was supported by the NSF Graduate Research Fellowship. Bernd Sturmfels was partially supported by NSF grant DMS-0456960. 
Let us begin by examining the simplest case: a parametrized curve in the plane.

Example 1.1. Let $d=1$ and $n=2$. We wish to compute the equation $g\left(x_{1}, x_{2}\right)$ of the plane algebraic curve parametrized by two Laurent polynomials $x_{1}=f_{1}(t)$ and $x_{2}=f_{2}(t)$. The Newton polytopes of $f_{1}$ and $f_{2}$ are segments on the line $\mathbb{R}^{1}$,

$$
P_{1}=[a, b] \text { and } P_{2}=[c, d] \text {. }
$$

Here $a \leq b$ and $c \leq d$ are integers. The Newton polygon $Q$ of the implicit equation $g\left(x_{1}, x_{2}\right)$ is a (possibly degenerate) quadrangle in $\mathbb{R}^{2}$. There are four cases:

- if $a \geq 0$ and $c \geq 0$ then $Q=\operatorname{conv}\{(0, b),(0, a),(c, 0),(d, 0)\}$,

- if $b \leq 0$ and $d \leq 0$ then $Q=\operatorname{conv}\{(0,-a),(0,-b),(-d, 0),(-c, 0)\}$,

- if $a \leq 0, d \geq 0$ and $b c \geq a d$ then $Q=\operatorname{conv}\{(0, b-a),(0,0),(d-c, 0),(d,-a)\}$,

- if $b \geq 0, c \leq 0$ and $b c \leq a d$ then $Q=\operatorname{conv}\{(0, b-a),(0,0),(d-c, 0),(-c, b)\}$.

The possible quadrangles $Q$ and their normal fans are illustrated in Figure 1 We note that the case $a=48, b=63, c=d=32$ appears in [EK Example 3.3].
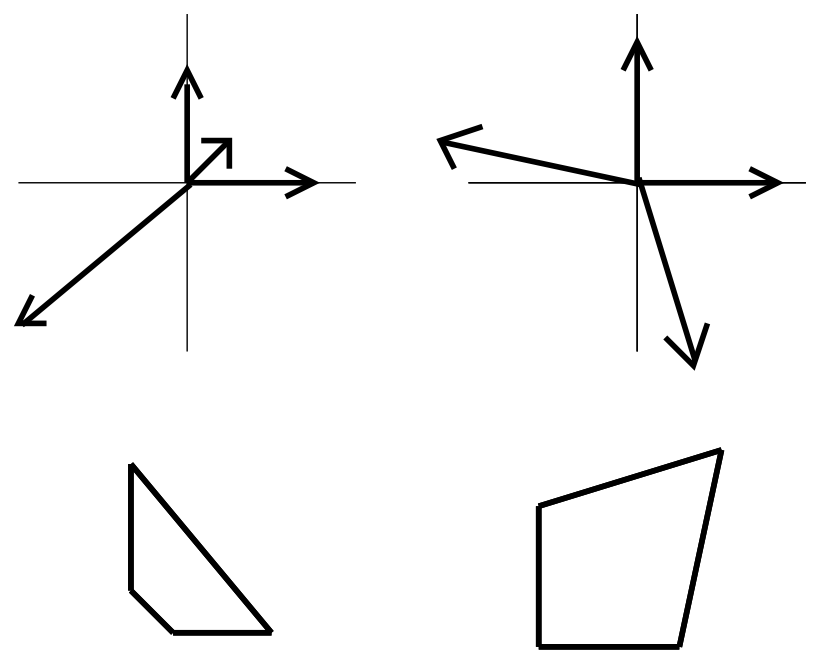

FIGURE 1. Tropical plane curves and their Newton polygons

Tropical implicitization can be visualized using Figure 1 Given the input (1.1), what we compute is the tropical curve $\mathcal{T}(g)$. This is the picture in the first row. By definition, $\mathcal{T}(g)$ is the set of rays in the inner normal fan of the (unknown) polygon in the second row. The output is the collection of four rays which are spanned by the vectors $(b-a, 0),(0, d-c),(a, c)$, and $(-b,-d)$. These vectors sum to zero, and they determine the polygon $Q$ according to the four cases spelled out above.

The motivation behind both $[\mathrm{EK}$ and $[\mathrm{SY}]$ is that a priori knowledge of the Newton polytope $Q$ would greatly facilitate the subsequent computation of recovering the coefficients of $g(x)$ from the coeffcients of $f_{1}(t), \ldots, f_{n}(t)$. This is a problem of numerical linear algebra, and it will be discussed in Subsection 5.2

Our presentation is organized into four sections as follows. In Section 2 we present our first main result, Theorem 2.1] which is a formula for the tropical variety $\mathcal{T}(I)$ in terms of the Newton polytopes $P_{1}, \ldots, P_{n}$. In Section 3 we demonstrate applications of this formula for a wide range of examples. In Section 4 we give a 
formula, in terms of mixed volumes and lattice indices, for the intrinsic multiplicities of the maximal cones in the tropical variety $\mathcal{T}(I)$. Knowledge of these multiplicities is essential when trying to reconstruct information about the ideal $I$ from its tropical variety $\mathcal{T}(I)$. In Section 5 we present our algorithm for computing the Newton polytope $Q$ of the implicit equation (or the Chow polytope of $I$ ) from $P_{1}, \ldots, P_{n}$.

\section{The tropical VARIETy of THE IMPLICIT EQUATIONS}

Our implicitization problem is specified by a collection of $n$ Laurent polynomials

$$
f_{i}(t)=\sum_{a \in A_{i}} c_{i, a} \cdot t_{1}^{a_{1}} \cdots t_{d}^{a_{d}} \quad(i=1,2, \ldots, n) .
$$

Here each $A_{i}$ is a finite subset of $\mathbb{Z}^{d}$, and the $c_{i, a}$ are generic complex numbers. Our ultimate aim is to compute the ideal $I \subset \mathbb{C}\left[x_{1}, \ldots, x_{n}\right]$ of algebraic relations among $f_{1}(t), \ldots, f_{n}(t)$, or at least, some information about its variety $V(I)$.

The tropical approach to this problem is based on the following idea. Rather than computing $V(I)$ by algebraic means, we shall compute the tropical variety $\mathcal{T}(I) \subset \mathbb{R}^{n}$ by combinatorial means. We recall (e.g. from BJSST PS, SS ) that the tropical variety $\mathcal{T}(I)$ is the set of all vectors $w \in \mathbb{R}^{n}$ such that the initial ideal $\operatorname{in}_{w}(I)=\left\langle\operatorname{in}_{w}(g): g \in I\right\rangle$ contains no monomials. Here $\operatorname{in}_{w}(g)$ is the initial form of $g$ which is the sum of all terms $c_{\alpha} x^{\alpha}$ in $g$ which have $w$-minimal weight $\alpha \cdot w$.

Let $\Psi: \mathbb{R}^{d} \rightarrow \mathbb{R}^{n}$ be the tropicalization of the map $f=\left(f_{1}, \ldots, f_{n}\right)$, i.e., let $\Psi_{i}(w)=\min \left\{w \cdot v: v \in P_{i}\right\}$ be the support function of the Newton polytope $P_{i}=\operatorname{conv}\left(A_{i}\right)$. The image of $\Psi$ is contained in the tropical variety $\mathcal{T}(I)$, by $[\mathrm{PS}$, Theorem 2], but this containment is usually strict. In other words, the image of the tropicalization of $f$ is usually a proper subset of the tropicalization of the image of $f$. The point of the following result is to characterize the difference $\mathcal{T}(I) \backslash$ image $(\Psi)$.

Let $e_{1}, \ldots, e_{n}$ be the standard basis of $\mathbb{R}^{n}$. For $J \subseteq\{1, \ldots, n\}$, we abbreviate the orthant $\mathbb{R}_{\geq 0}\left\{e_{j}: j \in J\right\}$ by $\mathbb{R}_{\geq 0}^{J}$ and the Minkowski sum $\sum_{j \in J} P_{j}$ by $P_{J}$.

Theorem 2.1. Let $f_{1}, \ldots, f_{n}$ be Laurent polynomials in $d$ variables which are generic relative to their support, and let $I \subset \mathbb{C}\left[x_{1}, \ldots, x_{n}\right]$ be the prime ideal of all algebraic relations among $f_{1}, \ldots, f_{n}$. Then the following subsets of $\mathbb{R}^{n}$ coincide:

(1) the tropical variety $\mathcal{T}(I)$,

(2) the union of all sets $\Psi\left(\mathcal{T}\left(\left\langle f_{j}: j \in J\right\rangle\right)\right)+\mathbb{R}_{\geq 0}^{J}$, where $J \subseteq\{1,2, \ldots, n\}$,

(3) the union of the cones $\Psi(w)+\mathbb{R}_{>0}^{J}$, where $w \in \mathbb{R}^{d}$ and $J$ runs over all subsets of $\{1,2, \ldots, n\}$ such that $\left\langle i_{w}\left(f_{j}\right): j \in J\right\rangle$ contains no monomial,

(4) the union of the cones $\Psi(w)+\mathbb{R}_{\geq 0}^{J}$ such that, for all subsets $K \subseteq J$, the linear form $w$ attains its minimum over $P_{K}$ at a face of dimension $\geq|K|$.

Characterization (4) gives rise to a combinatorial algorithm for computing the tropical variety $\mathcal{T}(I)$ directly from the given Newton polytopes $P_{1}, \ldots, P_{n}$. We will see examples in Section 3, and in Section 5 we discuss our implementation of this algorithm. In this section we explain Theorem 2.1 and give an outline of its proof.

It is instructive to note that the contribution of the empty set $J=\emptyset$ in Theorem 2.1 (2) is precisely the image of the tropicalization $\Psi$ of the given map $f$ :

$$
\Psi(\mathcal{T}(\langle\emptyset\rangle))+\mathbb{R}_{\geq 0}^{\emptyset}=\Psi(\mathcal{T}(\{0\}))=\Psi\left(\mathbb{R}^{d}\right)=\operatorname{image}(\Psi) .
$$

Thus it is the contributions made by non-empty subsets $J$ which make up the difference between the tropicalization of the image and the image of the tropicalization. 
Example 2.2. Consider the case of a plane curve as in Example 1.1 Assume for simplicity that $f_{1}$ and $f_{2}$ are not monomials, i.e., $a<b$ and $c<d$. For $J=\{1,2\}$ we just get the empty set since $\left\langle f_{1}, f_{2}\right\rangle$ is the unit ideal in $\mathbb{C}\left[t, t^{-1}\right]$. For $J=\{1\}$ we get the ray spanned by $(1,0)$ since $\mathcal{T}\left(\left\langle f_{1}\right\rangle\right)=\{0\}$, and for $J=\{2\}$ we get the ray spanned by $(0,1)$. Finally, for $J=\emptyset$ we get the image of the tropicalization $\Psi$ which consists of the two rays spanned by $(a, c)$ and by $(-b,-d)$. See Figure 1

We continue by explaining the meaning of the combinatorial conditions (2), (3) and (4), and, in the course of doing so, we shall prove that they are equivalent. First of all, we relabel so that $J=\{1, \ldots, r\}$ and consider the system of equations

$$
\operatorname{in}_{w}\left(f_{1}\right)(t)=\cdots=\operatorname{in}_{w}\left(f_{r}\right)(t)=0 .
$$

The Newton polytopes of these Laurent polynomials are face $_{w}\left(P_{1}\right), \ldots$, face $_{w}\left(P_{r}\right)$, where face $_{w}\left(P_{i}\right)$ is the face of $P_{i}$ at which the linear functional $u \mapsto u \cdot w$ attains its minimum. Let $s$ denote the dimension of the polytope

$$
\text { face }_{w}\left(P_{J}\right)=\text { face }_{w}\left(P_{1}\right)+\cdots+\text { face }_{w}\left(P_{r}\right)
$$

We wish to determine whether or not the system (2.3) has a solution $t$ in the algebraic torus $\left(\mathbb{C}^{*}\right)^{d}$. The equations (2.3) can be rewritten as a system of Laurent polynomials in $s$ unknowns. Since the coefficients of the $f_{i}$ are assumed to be generic, a necessary condition for (2.3) to be solvable is that $r \leq s$. Let us first consider the case $r=s$. By Bernstein's Theorem $\mathrm{Be}, \mathrm{Kho}$, the number of solutions to the system (2.3) equals

$$
\text { MixedVolume }\left(\operatorname{face}_{w}\left(P_{1}\right), \ldots, \text { face }_{w}\left(P_{r}\right)\right) \text {, }
$$

where the mixed volume is normalized with respect to the lattice parallel to the affine span of face $_{w}\left(P_{J}\right)$. In degenerate cases this mixed volume may be zero.

Lemma 2.3. Suppose $r=s$. Then the following conditions are equivalent:

(1) The mixed volume 2.5) is positive.

(2) The system (2.3) has at least one solution $t \in\left(\mathbb{C}^{*}\right)^{d}$.

(3) For all subsets $K \subseteq J$, the polytope face ${ }_{w}\left(P_{K}\right)$ has dimension $\geq|K|$.

Proof. The equivalence of (1) and (2) follows from Bernstein's Theorem $\mathrm{Be}$. The equivalence of (1) and (3) appears in [Ew, Theorem 4.13, page 127].

Next suppose that $r<s$. Then we can artificially add $s-r$ equations with generic coefficients to obtain a system of $s$ equations in $s$ unknowns. Application of Lemma 2.3 to this new system shows that the dimension criterion continues to hold. Furthermore, the following proposition shows that the solvability of (2.3) is equivalent to the conditions appearing in Theorem 2.1

Proposition 2.4. For any $w \in \mathbb{R}^{d}$ and $J=\{1, \ldots, r\}$ the following are equivalent:

(1) The initial system [2.3) has at least one solution $t \in\left(\mathbb{C}^{*}\right)^{d}$.

(2) For all subsets $K \subseteq J$, the polytope face $_{w}\left(P_{K}\right)$ has dimension at least $|K|$.

(3) The ideal $\left\langle i n_{w}\left(f_{j}\right): j \in J\right\rangle$ contains no monomial.

(4) The vector $w$ lies in $\mathcal{T}\left(\left\langle f_{j}: j \in J\right\rangle\right)$.

Proof. The equivalence of (1) and (2) follows from Lemma 2.3. Since monomials are units in the Laurent polynomial ring, Hilbert's Nullstellensatz shows that (1) and (3) are equivalent. Now (4) holds if and only if there is no monomial in $\operatorname{in}_{w}\left(\left\langle f_{1}, \ldots, f_{r}\right\rangle\right)$. This condition implies (3) but, a priori, it may be stronger. To 
see that they are equivalent, we will use the hypothesis that the coefficients of the $f_{i}$ are generic and show that (1) implies (4). Suppose that (1) holds, and let $t^{0} \in\left(\mathbb{C}^{*}\right)^{d}$ be a solution of the system of equations (2.3). Using the polyhedral homotopy of HS, we can construct a solution $t^{1}$ of the equations $f_{1}(t)=\cdots=f_{r}(t)=0$ which degenerates to $t^{0}$ under the one-parameter torus given by $w$. The deformation $t^{0} \rightarrow t^{1}$ ensures that $t^{0}$ is a point in the variety of the initial ideal $\operatorname{in}_{w}\left(\left\langle f_{1}, \ldots, f_{r}\right\rangle\right)$, which therefore contains no monomial.

Remark 2.5. Our deformation argument for (1) $\Longrightarrow$ (4) can be understood geometrically using the smooth projective toric variety $Y$ below. Namely, both $t^{0}$ and $t^{1}$ are points on $Y$ that lie in the intersection of divisors $\bar{E}_{1} \cap \cdots \cap \bar{E}_{r}$ but $t^{1}$ lies in the dense torus while $t^{0}$ lies in the toric boundary in direction $w$.

Proposition 2.4 shows that the last three conditions in Theorem 2.1] are equivalent. To make the connection to the first condition, we need a few ingredients from algebraic geometry. Let $E_{i}=\left\{t \in\left(\mathbb{C}^{d}\right)^{*}: f_{i}(t)=0\right\}$ and $Y^{0}=\left(\mathbb{C}^{*}\right)^{d} \backslash \bigcup_{i=1}^{n} E_{i}$. The Laurent polynomials (2.1) specify a morphism of affine algebraic varieties

$$
f: Y^{0} \rightarrow\left(\mathbb{C}^{*}\right)^{n}, \quad z \mapsto\left(f_{1}(z), \ldots, f_{n}(z)\right)
$$

Our goal is to compute the tropicalization of the image of $f$. Suppose that $Y \supset Y^{0}$ is any smooth compactification of $Y^{0}$ whose boundary $D=Y \backslash Y^{0}$ is a divisor with simple normal crossings. Let $D_{1}, \ldots, D_{m}$ denote the irreducible components of the boundary divisor $D$. Let $\Delta_{Y, D}$ denote the simplicial complex on $\{1, \ldots, m\}$ whose simplices are the indices of subsets of divisors $D_{i}$ whose intersection on $Y$ is nonempty. Since $D$ is a normal crossing divisor, the simplicial complex $\Delta_{Y, D}$ is pure of dimension $d-1$. For any of the irreducible divisors $D_{i}$, let $\left[D_{i}\right] \in \mathbb{Z}^{n}$ be the vector (val $D_{D_{i}} f_{1}, \ldots, \operatorname{val}_{D_{i}} f_{n}$ ), where $\operatorname{val}_{D_{i}}$ is the order of zeros-poles along $D_{i}$.

Theorem 2.6. The tropical variety $\mathcal{T}(I)$ is equal to the union of cones

$$
\mathcal{T}(I)=\bigcup_{\sigma \in \Delta_{Y, D}} \mathbb{R}_{\geq 0}\left\{\left[D_{i}\right]: i \in \sigma\right\} .
$$

The proof of this theorem will appear in [STY] and follows the work in [HKT]. There, $Y^{0}$ may be any variety that is very affine, which means that it is closed in a torus, and $f$ is allowed to be any morphism of very affine varieties. The required compactification $Y$ of the very affine variety $Y^{0}$ can be constructed, in principle, by appealing to Hironaka's Theorem on resolution of singularities. In practice, however, we are interested in situations where $Y$ is more readily available.

This is precisely the case studied in this paper, namely, when the coefficients $c_{i, a}$ of the Laurent polynomials $f_{i}(t)$ are assumed to be generic. In this case the desired compactification $Y$ of $Y^{0}$ is furnished to us by the methods of toric geometry.

Proof of Theorem 2.1. The equivalence of the sets in (2), (3) and (4) was shown in Proposition 2.4 What remains to be shown is the equivalence of (1) and (2).

Let $Y$ be a $d$-dimensional smooth projective toric variety whose polytope $\tilde{P}$ has the given Newton polytopes $P_{1}, \ldots, P_{n}$ as Minkowski summands. The smooth toric variety $Y$ is a compactification of $Y^{0}$. It comes with a canonical morphism $Y \rightarrow Y_{i}$ onto the (generally not smooth) toric variety $Y_{i}$ associated with $P_{i}$.

We claim that its boundary $Y \backslash Y^{0}$ has simple normal crossings. The irreducible components of $Y \backslash Y^{0}$ are of two types. Firstly, we have toric divisors $D_{1}, \ldots, D_{l}$ indexed by the facets of $\tilde{P}$. The toric boundary $D_{1} \cup \cdots \cup D_{l}$ of $Y$ has simple 
normal crossings because $\tilde{P}$ is a simple smooth polytope. Secondly, we have divisors $\bar{E}_{1}, \ldots, \bar{E}_{n}$ which are the closures in $Y$ of the divisors $E_{1}, \ldots, E_{n}$ in $\left(\mathbb{C}^{*}\right)^{d}$. The divisor $\bar{E}_{i}$ is the pullback under the morphism $Y \rightarrow Y_{i}$ of a general hyperplane section of the projective embedding of $Y_{i}$ defined by $P_{i}$. Bertini's Theorem implies that the $\bar{E}_{i}$ are smooth and irreducible, and that the union of all $D_{i}$ 's and all $\bar{E}_{j}$ 's has normal crossings. (Here we are tacitly assuming that each polytope $P_{i}$ has dimension $\geq 2$. If $\operatorname{dim}\left(P_{i}\right)=1$ then $\bar{E}_{i}$ is the disjoint union of smooth and irreducible divisors, and the following argument needs to be slightly modified).

In summary, we have that Theorem 2.6 can be applied to

$$
Y \backslash Y^{0}=D_{1} \cup D_{2} \cup \cdots \cup D_{l} \cup \bar{E}_{1} \cup \cdots \cup \bar{E}_{n} .
$$

The simplicial complex $\Delta_{Y, D}$ has dimension $d-1$ and it has $m=l+n$ vertices, one for each of the divisors $D_{i}$ and $\bar{E}_{j}$. Its maximal simplices correspond to pairs $(C, J)$ where $C=\left\{i_{1}, \ldots, i_{d-r}\right\} \subseteq\{1, \ldots, l\}$ and $J=\left\{j_{1}, \ldots, j_{r}\right\} \subseteq\{1, \ldots, n\}$ and

$$
D_{i_{1}} \cap \cdots \cap D_{i_{d-r}} \cap \bar{E}_{j_{1}} \cap \cdots \cap \bar{E}_{j_{r}} \neq \emptyset .
$$

For any $J \subseteq\{1, \ldots, n\}$ let $\Delta_{Y, D}[J]$ denote the subset of $\Delta_{Y, D}$ consisting of all simplices with fixed $J$. Note that $\Delta_{Y, D}[\emptyset]$ is the boundary complex of the simplicial polytope dual to $\tilde{P}$. Moreover, $\Delta_{Y, D}[J]=\{\emptyset\}$ if $|J|=d$, and $\Delta_{Y, D}[J]=\emptyset$ if $|J|>d$. For each $j \in J$ we have $\left[\bar{E}_{j}\right]=\left(\operatorname{val}_{\bar{E}_{j}} f_{1}, \ldots, \operatorname{val}_{\bar{E}_{j}} f_{n}\right)=e_{j}$ is the $j^{\text {th }}$ basis vector in $\mathbb{R}^{n}$. Hence the formula [2.7] in Theorem [2.6] can be rewritten as follows:

$$
\mathcal{T}(I)=\bigcup_{J \subset\{1, \ldots, n\}}\left(\mathbb{R}_{\geq 0}^{J}+\bigcup_{C \in \Delta_{Y, D}[J]} \mathbb{R}_{\geq 0}\left\{\left[D_{i}\right]: i \in C\right\}\right) .
$$

Hence to prove the remaining equivalence $(1)=(2)$, it suffices to show that

$$
\Psi\left(\mathcal{T}\left(\left\langle f_{j}: j \in J\right\rangle\right)\right)=\bigcup_{C \in \Delta_{Y, D}[J]} \mathbb{R}_{\geq 0}\left\{\left[D_{i}\right]: i \in C\right\} .
$$

Let $w_{i} \in \mathbb{R}^{d}$ denote the primitive inner normal vector of the facet of the polytope $\tilde{P}$ corresponding to the divisor $D_{i}$. For any $j \in J$, the integer $\operatorname{val}_{D_{i}}\left(f_{j}\right)$ is the value at $w_{i}$ of the support function of the polytope $P_{j}$. Hence $\left[D_{i}\right]=\Psi\left(w_{i}\right)$ in $\mathbb{R}^{n}$, so right hand side of (2.9) is the image under $\Psi$ of the subfan of the normal fan of $\tilde{P}$ indexed by $\Delta_{Y, D}[J]$. Thus it suffices to show that this subfan coincides with $\mathcal{T}\left(\left\langle f_{j}: j \in J\right\rangle\right)$. A vector $w$ lies in this subfan if and only if 2.8 holds, which is the same as saying that the system $\operatorname{in}_{w}\left(f_{j}\right)=0$ for $j \in J$ has a solution in $\left(\mathbb{C}^{*}\right)^{d}$. By Proposition 2.4 this is equivalent to $w \in \mathcal{T}\left(\left\langle f_{j}: j \in J\right\rangle\right)$. This completes our proof of Theorem 2.1]

\section{EXAMPLES}

We now present five families of examples which illustrate the Theorem 2.1

3.1. Monomials. Suppose that each $f_{i}$ is a monomial, so each $P_{i}$ is just a point, say $P_{i}=\left\{a_{i}\right\} \subset \mathbb{Z}^{d}$. Then $I$ is the toric ideal generated by all binomials $x^{u}-x^{v}$ where $u-v$ is in the kernel of the matrix $A=\left[a_{1}, a_{2}, \ldots, a_{n}\right]$. The tropical variety $\mathcal{T}(I)$ equals the row space of $A$. The tropical morphism $\Psi: \mathbb{R}^{d} \rightarrow \mathbb{R}^{n}$ is the linear map $w \mapsto w A$ given by left multiplication with $A$. For any non- empty subset $J$ of $\{1,2, \ldots, n\}$, the ideal $\left\langle f_{j}: j \in J\right\rangle$ is the unit ideal, which contributes nothing to 
the union in (2) of Theorem 2.1] The ideal corresponding to $J=\emptyset$ is $\langle\emptyset\rangle=\{0\}$, and $\mathcal{T}(I)=\operatorname{rowspace}(A)$ is indeed the image of $\mathcal{T}(\langle\emptyset\rangle)=\mathbb{R}^{d}$ under the map $\Psi$.

3.2. The Unmixed Case. Suppose that all Newton polytopes are equal, say $P_{1}=$ $\ldots=P_{n}=P$, and $P$ has the maximal dimension $d$. We distinguish two cases.

First suppose that $P$ contains the origin. Then the image of $\Psi$ is the halfline $C^{-}=\mathbb{R}_{\leq 0}\left(e_{1}+\ldots+e_{n}\right)$. In fact, the set $\Psi\left(\mathcal{T}\left(\left\langle f_{j}: j \in J\right\rangle\right)\right)$ in Theorem 2.1 (2) equals $C^{-}$if $|J|<d$, it equals $\{0\}$ if $|J|=d$, and it is empty if $|J|>d$. Therefore, the tropical variety $\mathcal{T}(I)$ is the union of the cones $\mathbb{R}_{\geq 0}^{J}$ for $J \subset\{1, \ldots, n\}$ with $|J|=d$ and the cones $C^{-}+\mathbb{R}_{\geq 0}^{J}$ for $J \subset\{1, \ldots, n\}$ with $|J|=d-1$.

Next suppose that $P$ does not contain the origin. Then the image of $\Psi$ is the line $C=\mathbb{R}\left(e_{1}+\ldots+e_{n}\right)$, and we conclude that the tropical variety $\mathcal{T}(I)$ is the union of the cones $\mathbb{R}_{\geq 0}^{J}$ for $|J|=d$, and the cones $C+\mathbb{R}_{\geq 0}^{J}$ for $|J|=d-1$.

In the case $n=d+1$, the tropical variety $\mathcal{T}(I)$ just constructed has codimension one in $\mathbb{R}^{n}$. It is the union of all codimension one cones in the normal fan of the Newton polytope $Q$ of the implicit equation. The polytope $Q$ was constructed with a different technique in [SY] Theorem 9], and the previous two paragraphs are consistent with that result. However, knowledge of $\mathcal{T}(I)$ is not sufficient to determine $Q$. For that we need the formula for multiplicities in Section 4 below.

Example 3.1. Let $P$ be any convex lattice polygon in $\mathbb{R}^{2}$ and consider three generic bivariate Laurent polynomials with Newton polygon $P$. Then $\mathcal{T}(I)$ is a tropical surface in $\mathbb{R}^{3}$. We abbreviate $e_{4}=-e_{1}-e_{2}-e_{3}$. If the polygon $P$ contains the origin in $\mathbb{R}^{2}$, then $\mathcal{T}(I)$ is the fan consisting of the six two-dimensional cones

$$
\mathbb{R}_{\geq 0}\left\{e_{1}, e_{2}\right\}, \mathbb{R}_{\geq 0}\left\{e_{1}, e_{3}\right\}, \mathbb{R}_{\geq 0}\left\{e_{2}, e_{3}\right\}, \mathbb{R}_{\geq 0}\left\{e_{1}, e_{4}\right\}, \mathbb{R}_{\geq 0}\left\{e_{2}, e_{4}\right\}, \mathbb{R}_{\geq 0}\left\{e_{3}, e_{4}\right\} .
$$

This implies that $Q$ is a tetrahedron $\operatorname{conv}\left\{0, \alpha e_{1}, \alpha e_{2}, \alpha e_{3}\right\}$, for some $\alpha>0$.

If $P$ does not contain the origin, then the tropical surface $\mathcal{T}(I)$ has a natural fan structure which is given by the nine two-dimensional cones

$$
\mathbb{R}_{\geq 0}\left\{e_{i}, e_{j}\right\}, \mathbb{R}_{\geq 0}\left\{e_{i}, e_{4}\right\}, \mathbb{R}_{\geq 0}\left\{e_{i},-e_{4}\right\} \quad(1 \leq i<j \leq 3) .
$$

This implies that $Q$ is a triangular prism $\operatorname{conv}\left\{\alpha e_{1}, \alpha e_{2}, \alpha e_{3}, \beta e_{1}, \beta e_{2}, \beta e_{3}\right\}$, for some $\alpha>\beta>0$. The parameters $\alpha, \beta$ are expressed in terms of volumes as in [SY] Theorem 9]. Alternatively, we can determine $\alpha$ and $\beta$ using Theorem 4.1 below.

3.3. Linear maps. Suppose each support $A_{i}$ is a subset of $\left\{e_{1}, \ldots, e_{d}\right\}$, the unit vectors in $\mathbb{R}^{d}$. Thus $f_{i}$ is a generic linear form in the set of unknowns $\left\{t_{j}\right.$ : $\left.e_{j} \in A_{i}\right\}$. The ideal $I$ is generated by an $(n-d)$-dimensional space of linear forms in $\mathbb{C}\left[x_{1}, \ldots, x_{n}\right]$. We introduce the subset $B_{j}=\left\{i: e_{j} \in A_{i}\right\}$ for $j=$ $1,2, \ldots, d$. The rank $d$ matroid corresponding to the linear space $V(I) \subset \mathbb{C}^{n}$ is the transversal matroid $\left[\mathrm{Br}\right.$ of the set family $\left\{B_{1}, \ldots, B_{d}\right\}$. The tropical variety $\mathcal{T}(I)$ is the Bergman complex of that transversal matroid, as shown in AK.

Thus, in the linear case, Theorem 2.1 offers an interesting new representation of the Bergman fans of transversal matroids. It can be described as follows. The sum of simplices $P=\sum_{i=1}^{n} \operatorname{conv}\left(A_{i}\right)$ is a generalized permutohedron $[\mathrm{Po}$, which means that the normal fan of $P$ is a coarsening of the $S_{n}$-arrangement. Let $C$ be any cone in that fan. Each initial form $\operatorname{in}_{C}\left(f_{j}\right)$ is supported on a subset face ${ }_{C}\left(A_{j}\right)$ of $A_{j}$. Then with $C$ we associate the simplicial complex $\Delta_{C}$ on $\{1,2, \ldots, n\}$ whose simplices are the subsets $J$ such that these initial forms have a solution in $\left(\mathbb{C}^{*}\right)^{d}$. This is a combinatorial condition on the set family $\left\{\operatorname{face}_{C}\left(A_{j}\right): j \in J\right\}$, which 
is essentially Postnikov's Dragon Marriage Condition [Po $\$$ \$5]. To construct the Bergman fan, we map the normal fan of $P$ from $\mathbb{R}^{d}$ into $\mathbb{R}^{n}$ using the map $\Psi$, and to each image cone $\Psi(C)$ we attach the family of orthants $\mathbb{R}_{\geq 0}^{J}$ indexed by $\Delta_{C}$.

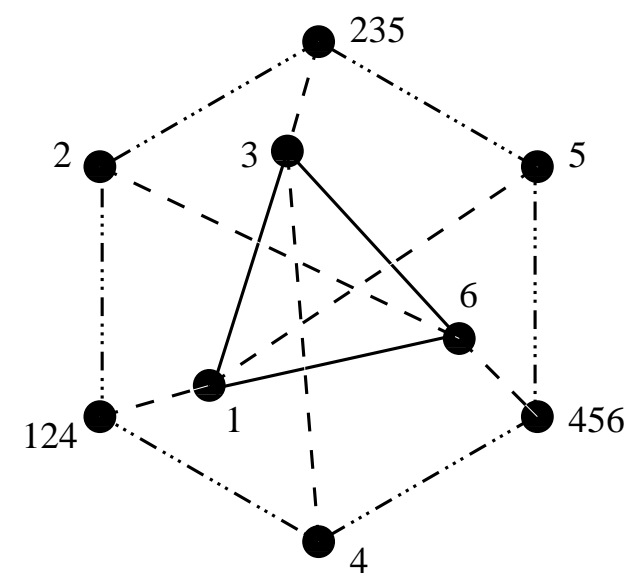

Figure 2. The Bergman complex of a transversal matroid

Example 3.2. Let $d=3, n=6$ and consider six linear forms

$$
\begin{array}{llc}
f_{1}= & c_{11} \cdot t_{1}+c_{12} \cdot t_{2} \\
f_{2}= & c_{21} \cdot t_{1} \\
f_{3}= & c_{31} \cdot t_{1}+c_{33} \cdot t_{3} \\
f_{4}= & c_{42} \cdot t_{2} \\
f_{5}= & c_{53} \cdot t_{3} \\
f_{6}= & c_{62} \cdot t_{2}+c_{63} \cdot t_{3},
\end{array}
$$

where the $c_{i j}$ are general complex numbers. The tropical linear space $\mathcal{T}(I)$ is three-dimensional in $\mathbb{R}^{6}$, but each maximal cone contains the line spanned by $(1,1,1,1,1,1)$, so we can represent $\mathcal{T}(I)$ by a graph. This graph is the Bergman complex of a rank 3 matroid on $\{1,2,3,4,5,6\}$, namely, the transversal matroid of

$$
\left(B_{1}, B_{2}, B_{3}\right)=(\{1,2,3\},\{1,4,6\},\{3,5,6\}) .
$$

The drawing of $\mathcal{T}(I)$ in Figure 2 is the same as [DFS, Figure 2], but we now derive it from Theorem 2.1 (2). The polytope $P=P_{1}+\cdots+P_{6}$ is a planar hexagon, and $\Psi$ maps the six two-dimensional cones in its normal fan to the six edges in the outer hexagon in Figure 2 Each of the edges $\{124,1\},\{2,6\},\{235,3\},\{5,1\},\{456,6\}$, and $\{4,3\}$ are between the image of a ray in the normal fan of $P$ and a ray generated by $e_{1}, e_{3}$, or $e_{6}$. For instance, the edge $\{124,1\}$ corresponds to the ray generated by $\Psi((1,1,0))=(1,1,0,1,0,0)$ plus the ray $\mathbb{R}_{\geq 0} e_{1}$ in $\mathbb{R}^{6}$. None of the simplicial complexes $\Delta_{C}$ contains 2,4 , or 5 because $f_{2}, f_{4}$, and $f_{5}$ are monomials. However, $e_{2}, e_{4}$, and $e_{5}$ are images of some rays in the normal fan of $P$ under the tropical map $\Psi$. The triangle in the middle of Figure 2 represents the simplicial complex associated with the 0 -dimensional face $\{0\}$ of the normal fan:

$$
\Delta_{\{0\}}=\{\{1,3\},\{1,6\},\{3,6\}\} .
$$

This shows how tropical implicitization works for the linear forms $f_{1}, \ldots, f_{6}$. 
3.4. Binomials. Consider the case where the map $f$ is given by $n$ binomials

$$
f_{i}=c_{i 1} \cdot t^{a_{i}}+c_{i 2} \cdot t^{b_{i}} \quad(i=1,2, \ldots, n) .
$$

Each Newton polytope $P_{i}=\operatorname{conv}\left(a_{i}, b_{i}\right)$ is a line segment, and their Minkowski sum $P=P_{1}+\cdots+P_{n}$ is a zonotope (i.e. a projection of the $n$-cube). The normal fan of $P$ is the hyperplane arrangement $\mathcal{H}=\left\{\left\{u \cdot a_{j}=u \cdot b_{j}\right\}\right\}_{j=1, \ldots, n}$. The map $\Psi: \mathbb{R}^{d} \rightarrow \mathbb{R}^{n}$ is the tropical morphism associated with the arrangement $\mathcal{H}$, that is,

$$
\Psi(u)=\left(\min \left(u \cdot a_{1}, u \cdot b_{1}\right), \min \left(u \cdot a_{2}, u \cdot b_{2}\right), \ldots, \min \left(u \cdot a_{n}, u \cdot b_{n}\right)\right) .
$$

This map was recently studied by Ardila $\mathrm{Ar}$ for certain graphic arrangements $\mathcal{H}$.

Theorem 2.1 shows that the tropical variety $\mathcal{T}(I)$ is the union of the cones $\Psi(C)+$ $\mathbb{R}_{>0}^{J}$, where $C$ is any cone of the hyperplane arrangement $\mathcal{H}$, and the hyperplanes indexed by $J \subset\{1, \ldots, n\}$ contain the cone $C$ and are linearly independent.

Example 3.3. Let $d=2$ and consider the following $n=3$ binomials in $t_{1}$ and $t_{2}$ :

$$
f_{1}=\left(t_{1}-\alpha\right) \cdot t_{1}^{u_{1}} t_{2}^{u_{2}}, f_{2}=\left(t_{2}-\beta\right) \cdot t_{1}^{v_{1}} t_{2}^{v_{2}}, f_{3}=\left(t_{1}-\gamma t_{2}\right) \cdot t_{1}^{w_{1}} t_{2}^{w_{2}},
$$

where $\alpha, \beta, \gamma$ are general complex numbers, and $u_{1}, u_{2}, v_{1}, v_{2}, w_{1}, w_{2}$ are integers. Then $\mathcal{H}$ is the arrangement of three lines in the plane $\mathbb{R}^{2}$ which consists of the two coordinate axes and the main diagonal. The images of the six one-dimensional cones of the arrangement $\mathcal{H}$ under the map $\Psi$ are spanned by the vectors

$$
\begin{aligned}
\Psi\left(e_{1}\right) & =\left(u_{1}, v_{1}, w_{1}\right), \\
\Psi\left(e_{1}+e_{2}\right) & =\left(u_{1}+u_{2}, v_{1}+v_{2}, w_{1}+w_{2}+1\right), \\
\Psi\left(e_{2}\right) & =\left(u_{2}, v_{2}, w_{2}\right), \\
\Psi\left(-e_{1}\right) & =\left(-u_{1}-1,-v_{1},-w_{1}-1\right), \\
\Psi\left(-e_{1}-e_{2}\right) & =\left(-u_{1}-u_{2}-1,-v_{1}-v_{2}-1, w_{1}-w_{2}-1\right), \\
\Psi\left(-e_{2}\right) & =\left(-u_{2},-v_{2}-1,-w_{2}-1\right) .
\end{aligned}
$$

The image of $\Psi$ consists of the six two-dimensional cones in $\mathbb{R}^{3}$ which are spanned by (cyclically) consecutive vectors in this list. Next, the tropical surface $\mathcal{T}(I)$ contains the six two-dimensional cones which are spanned by the following pairs in $\mathbb{R}^{3}$ :

$$
\begin{gathered}
\left\{\Psi\left(e_{1}\right), e_{2}\right\},\left\{\Psi\left(e_{1}+e_{2}\right), e_{3}\right\},\left\{\Psi\left(e_{2}\right), e_{1}\right\}, \\
\left\{\Psi\left(-e_{1}\right), e_{2}\right\},\left\{\Psi\left(-e_{1}-e_{2}\right), e_{3}\right\},\left\{\Psi\left(-e_{2}\right), e_{1}\right\} .
\end{gathered}
$$

Finally, the zero-dimensional cone $C=\{0\}$ contributes the three two-dimensional cones which are spanned by the pairs of standard basis vectors

$$
\left\{e_{1}, e_{2}\right\},\left\{e_{1}, e_{3}\right\},\left\{e_{2}, e_{3}\right\} .
$$

These pairs determine a non-planar graph with 9 vertices and 15 edges. It is isomorphic to the one depicted in Figure 2 Thus $\mathcal{T}(I)$ is a two-dimensional fan in $\mathbb{R}^{3}$ whose intersection with the unit sphere is an immersion of that graph. The resulting embedded graph, which is dual to the Newton polytope of the implicit equation, depends on the numerical values of the exponents $u_{1}, u_{2}, v_{1}, v_{2}, w_{1}, w_{2}$. This example shows how our method can be applied to a family of implicitization problems where the exponents of the $f_{i}$ are not fixed integers but are unknowns. 
3.5. Surfaces. Suppose that $d=2$, so our given input is a list of lattice polygons $P_{1}, \ldots, P_{n}$ in $\mathbb{R}^{2}$. The tropical variety $\mathcal{T}(I)$ is a two-dimensional fan in $\mathbb{R}^{n}$ which we represent by an embedded graph in the $(n-1)$-sphere $\mathbb{S}^{n-1}$. This graph is constructed as follows. We fix the node $e_{i}$ for each polygon $P_{i}$ which is not just a point, and we fix the node $\Psi(w)$ for each vector $w$ that is an inner normal to an edge of the polygon $P=P_{1}+\cdots+P_{n}$ and such that $\Psi(w) \neq 0$. We identify such a vector $\Psi(w)$ with the point $\Psi(w) /\|\Psi(w)\|$ on $\mathbb{S}^{n-1}$.

We now connect pairs of nodes by great circles in $\mathbb{S}^{n-1}$ according to the following rules: a pair $\left\{\Psi(w), \Psi\left(w^{\prime}\right)\right\}$ gets connected if their edges on $P$ are adjacent, a pair $\left\{\Psi(w), e_{i}\right\}$ gets connected if $w$ is the inner normal to an edge of $P_{i}$, and a pair $\left\{e_{i}, e_{j}\right\}$ gets connected if $P_{i}+P_{j}$ is two-dimensional. Some pairs of great circles intersect and thus create new nodes in $\mathbb{S}^{n-1}$. This intersection need not be transversal, i.e., two great circles may intersect in a smaller great circle. The result of this construction is the tropical surface $\mathcal{T}(I)$, represented by a graph in $\mathbb{S}^{n-1}$.

Example 3.4. Consider the family of surfaces in 3-space which is given by

$A_{1}=\{(1,0),(3,1),(2,2)\}, A_{2}=\{(-1,0),(0,-1),(0,0)\}, A_{3}=\{(2,1),(0,2),(1,3)\}$.

To construct the graph $\mathcal{T}(I)$ on the sphere $\mathbb{S}^{2}$, we first draw the three nodes $e_{1}, e_{2}, e_{3}$ and the nine nodes $\Psi(w)$ which are the images under $\Psi$ of the inner normals of the 9-gon $P_{1}+P_{2}+P_{3}$. These nine directions are given by the columns of the matrix

$$
\left.\begin{array}{rrrrrrrrr}
e_{2} & e_{3} & e_{1} & e_{2} & e_{3} & e_{1} & e_{2} & e_{3} & e_{1} \\
1 & 1 & -1 & -3 & -7 & -4 & -2 & 0 & 2 \\
-1 & -2 & -2 & 0 & 0 & 0 & 0 & -1 & -2 \\
2 & 4 & 0 & -2 & -5 & -4 & -3 & -2 & -2
\end{array}\right)
$$

We connect these twelve nodes with great circles as described above, namely, by forming the 9-cycle of these columns, by connecting them to the $e_{i}$ as indicated, and by forming the triangle $e_{1}, e_{2}, e_{3}$. This creates an embedded graph in $\mathbb{S}^{2}$ which has 14 vertices, 27 edges and 15 regions. The Newton polytope $Q$ of the implicit equation is dual to this graph, so it has 15 vertices, 27 edges and 14 facets. Using the methods to be described in the next two sections, we construct $Q$ metrically, and we find that $g\left(x_{1}, x_{2}, x_{3}\right)$ is a polynomial of degree 14 having 154 terms.

\section{Multiplicities}

Let $K=\mathbb{C}\left\{\left\{\epsilon^{\mathbb{R}}\right\}\right\}$ be the field of Puiseux series in the unknown $\epsilon$ which have real exponents and complex coefficients. The field $K$ is algebraically closed, and it has the surjective non-archimedean valuation

$$
\text { order }: K^{*} \rightarrow \mathbb{R}, \alpha \epsilon^{u}+\cdots \mapsto u \text {. }
$$

Hence, $\operatorname{order}(u)$ is the exponent of the $\epsilon$-monomial of lowest degree which appears with non-zero coefficient in the Puiseux series $u=u(\epsilon)$. The order map extends to vectors of length $n$ by coordinatewise application:

$$
\text { order }:\left(K^{*}\right)^{n} \rightarrow \mathbb{R}^{n},\left(u_{1}, \ldots, u_{n}\right) \mapsto\left(\operatorname{order}\left(u_{1}\right), \ldots, \operatorname{order}\left(u_{n}\right)\right) \text {. }
$$

For any ideal $I \subset \mathbb{C}\left[x_{1}, \ldots, x_{n}\right]$ we write $V(I) \subset\left(K^{*}\right)^{n}$ for the variety defined by $I$ in the algebraic torus over the Puiseux series field $K$. It is known (see e.g. [SS] that the tropical variety equals the image of the variety under the order map:

$$
\mathcal{T}(I)=\operatorname{order}(V(I))
$$


If $I$ is a prime ideal of dimension $d$, which we may assume in our implicitization problem, then $\mathcal{T}(I)$ has the structure of a pure $d$-dimensional polyhedral fan. This fan structure is non-unique, and it can derived by restricting the Gröbner fan of any homogenization of $I$. See BJSST for software and mathematical details.

Every maximal cone $\Gamma$ of the fan $\mathcal{T}(I)$ naturally comes with a multiplicity, which is a positive integer. The multiplicity $M_{\Gamma}$ of a $d$-dimensional cone $\Gamma$ is the sum of multiplicities of all monomial-free minimal associate primes of the initial ideal $\operatorname{in}_{v}(I)$ in $\mathbb{C}\left[x_{1}, \ldots, x_{n}\right]$ where $v \in \mathbb{R}^{n}$ is any point in the relative interior of the cone $\Gamma$. The multiplicities $M_{\Gamma}$ are an important piece of data which must also be determined when computing a tropical variety $\mathcal{T}(I)$. It is with these multiplicities that a tropical variety satisfies the balancing condition which ensures that tropical intersection numbers are independent of choices; see [Mi §3] and [Ka, §6.4].

We now consider the prime ideal $I$ of algebraic relations among the generic Laurent polynomials $f_{1}(t), \ldots, f_{n}(t)$. Generators for the ideal $I$ are (still) unknown, but in Theorem 2.1 we computed its tropical variety $\mathcal{T}(I)$ in combinatorial terms. In what follows we similarly compute the multiplicity $M_{\Gamma}$ for every maximal cone $\Gamma$ in a fan structure on $\mathcal{T}(I)$. Recall that $\mathcal{T}(I)$ is the union of cones of the form

$$
\Psi(C)+\mathbb{R}_{\geq 0}^{J},
$$

where $C$ is a cone of the normal fan of $P=P_{1}+\ldots+P_{n}$ and $\operatorname{dim}_{\text {face }}\left(P_{K}\right) \geq|K|$ for any subset $K \subseteq J$ for some (hence any) vector $w$ in the relative interior of $C$.

The cones in (4.2) do not generally form a fan, and they need to be subdivided to give a fan structure on the tropical variety $\mathcal{T}(I)$. We choose a fine enough fan structure on $\mathcal{T}(I)$ so that any cone (4.2) is the union of some cones of $\mathcal{T}(I)$.

We say that a pair $(C, J)$ covers a $d$-dimensional cone $\Gamma$ in $\mathcal{T}(I)$ if (4.2) is $d$ dimensional and contains $\Gamma$. There may be more than one pair $(C, J)$ which covers a fixed cone $\Gamma$. Suppose that $(C, J)$ covers $\Gamma$. Then the sublattice generated by $\Psi\left(C \cap \mathbb{Z}^{d}\right)+\mathbb{Z}^{J}$ has rank $d$ in $\mathbb{Z}^{n}$. We define index $(C, J)$ to be the index of that sublattice in the maximal rank $d$ sublattice of $\mathbb{Z}^{n}$ that contains it.

Let $F_{j}=$ face $_{w}\left(P_{j}\right)$ and $F_{J}=$ face $_{w}\left(\sum_{j \in J} P_{j}\right)=\sum_{j \in J} F_{j}$ for some (hence any) vector $w$ in the relative interior of $C$. Then the $|J|$-dimensional mixed volume

$$
\operatorname{MixedVolume}\left(F_{j}: j \in J\right)
$$

is exactly the same as the one in equation (2.5). This mixed volume is normalized with respect to the affine lattice spanned by the $|J|$-dimensional polytope $F_{J}$. Multiplying (4.3) by index $(C, J)$ we obtain the scaled mixed volume

$$
\operatorname{MixedVolume}\left(F_{j}: j \in J\right) \cdot \operatorname{index}(C, J)
$$

The following theorem characterizes the multiplicities of our tropical variety.

Theorem 4.1. The multiplicity $M_{\Gamma}$ of a maximal cone $\Gamma$ in the fan structure on $\mathcal{T}(I)$ is the sum of all scaled mixed volumes (4.4) where the pair $(C, J)$ covers $\Gamma$.

The proof of this theorem will be presented in [STY], along with the following

Remark 4.2. Theorems 2.1 and 4.1 remain valid, with the word "cone" replaced by the word "polyhedron", when the coefficients of the given Laurent polynomials $f_{1}, \ldots, f_{n}$ are no longer complex numbers but lie in the Puiseux series field $K$.

To illustrate Theorem 4.1 suppose that $d=2$ and consider the tropical surface $\mathcal{T}(I)$. It is represented by a graph as in Subsection 3.5. The edges of this graph 
are weighted with multiplicities as follows. The weight of a pair $\left\{\Psi(w), \Psi\left(w^{\prime}\right)\right\}$ is the greatest common divisor of the $2 \times 2$-minors of the $n \times 2$-matrix $\left(\Psi(w), \Psi\left(w^{\prime}\right)\right)$, divided by the determinant of the $2 \times 2$-matrix $\left(w, w^{\prime}\right)$. The weight of a pair $\left\{\Psi(w), e_{i}\right\}$ is the normalized length of the edge face ${ }_{w}\left(P_{i}\right)$ of $P_{i}$ times the greatest common divisor of all coordinates of $\Psi(w)$ but the $i^{\text {th }}$. The weight of a pair $\left\{e_{i}, e_{j}\right\}$ is the mixed area

$$
\text { MixedVolume }\left(P_{i}, P_{j}\right)=\operatorname{area}\left(P_{i}+P_{j}\right)-\operatorname{area}\left(P_{i}\right)-\operatorname{area}\left(P_{j}\right) .
$$

Now, when forming the embedded graph $\mathcal{T}(I) \subset \mathbb{S}^{n-1}$, each edge $\Gamma$ may be covered by more than one of the great circles created by these pairs, and we take $M_{\Gamma}$ to be the sum of their weights. With these multiplicities, the graph $\mathcal{T}(I)$ is balanced. Here is a concrete numerical example, taking from the computer algebra literature.
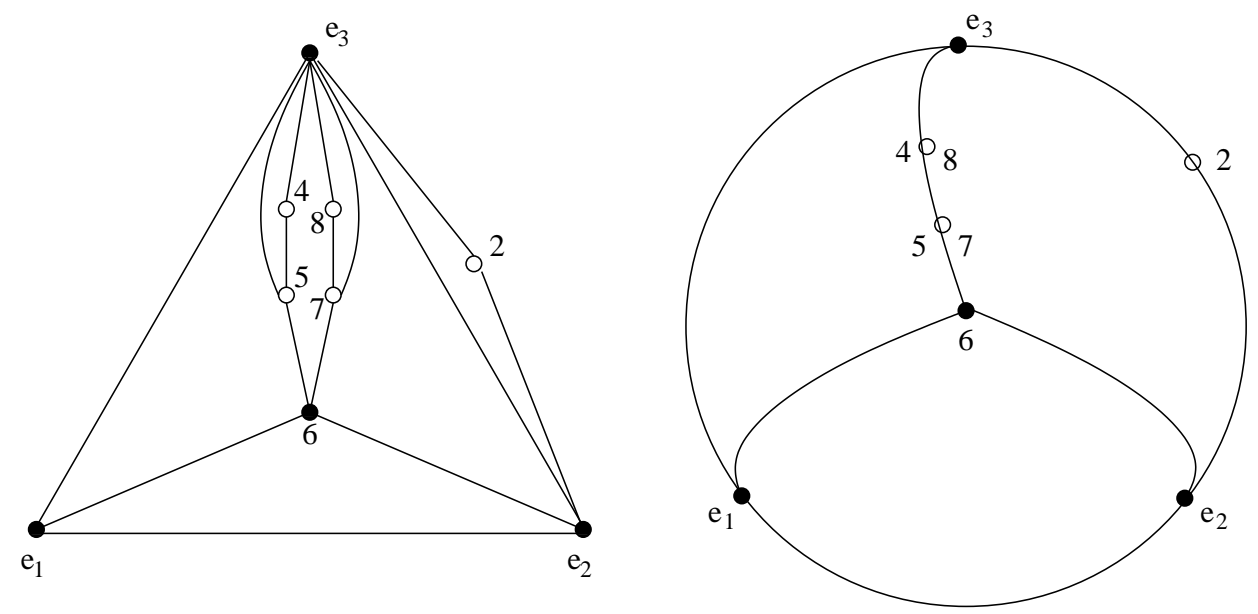

Figure 3. Tropical construction of a bicubic surface in 3-space

Example 4.3. Let $d=2$ and consider the well-known problem of implicitizing bicubic surfaces. The following specific surface was discussed in [EK] Example 3.4]:

$$
\begin{aligned}
f_{1}(s, t)= & 3 \mathbf{t}^{3}-6 t^{2}+3 t+\mathbf{s}^{3}-3 s^{2}+6 s-\mathbf{1} \\
f_{2}(s, t)= & 3 \mathbf{s}^{3}-6 s^{2}+3 \mathbf{s}+\mathbf{t}^{3}+3 \mathbf{t} \\
f_{3}(s, t)= & -3 \mathbf{s}^{3} \mathbf{t}^{3}+15 s^{2} t^{3}-15 \mathbf{s t}^{3}-3 s^{3} t^{2}-18 s^{2} t^{2}+27 s t^{2} \\
& -3 \mathbf{t}^{2}+6 \mathbf{s}^{3} \mathbf{t}+9 s^{2} t-18 s t+3 \mathbf{t}-3 \mathbf{s}^{2}+3 \mathbf{s} .
\end{aligned}
$$

\begin{tabular}{|c|c|c|c|c|c|c|c|c|}
\hline & $e_{1} e_{2} e_{3}$ & $e_{2} e_{3}$ & $e_{1} e_{2} e_{3}$ & $e_{3}$ & $e_{3}$ & $e_{1} e_{2}$ & $e_{3}$ & $e_{2}$ \\
\hline$w_{1}$ & 1 & 1 & 0 & -1 & -1 & -1 & 0 & \\
\hline$w_{2}$ & 0 & 1 & 1 & 1 & 0 & -1 & -1 & -1 \\
\hline$\Psi(w)_{1}$ & 0 & 0 & 0 & -3 & -3 & -3 & -3 & -3 \\
\hline$\Psi(w)_{2}$ & 0 & 1 & 0 & -3 & -3 & -3 & -3 & -3 \\
\hline$\Psi(w)_{3}$ & 0 & 1 & 0 & -2 & -3 & -6 & -3 & -2 \\
\hline
\end{tabular}

The vertices of the Newton polygons are indicated in boldface font. The polygon $P_{1}$ is a triangle, $P_{2}$ is a quadrangle, and $P_{3}$ is a 7 -gon. Their Minkowski sum $P_{1}+P_{2}+P_{3}$ is an octagon. The eight primitive inner normal vectors $w=\left(w_{1}, w_{2}\right)$ of this octagon are listed in the first two rows of the following matrix: 
The last three rows contain the coordinates of their images $\Psi(w)$ under the tropical morphism $\Psi$. We now construct the graph as in Subsection 3.5. The first and third inner normal vector is mapped to zero under $\Psi$, so they do not contribute to the tropical surface $\mathcal{T}(I)$. The remaining six columns $2,4,5,6,7,8$ and the unit vectors $e_{1}, e_{2}, e_{3}$ form the following 15 great circles on the sphere $\mathbb{S}^{2}$, as shown in Figure 3

$\begin{array}{cccc}\text { pair } & \text { index } & \text { mixed volume } & \text { on edge } \\ \left\{e_{1}, e_{2}\right\} & 1 & 9 & \left\{e_{1}, e_{2}\right\} \\ \left\{e_{1}, e_{3}\right\} & 1 & 18 & \left\{e_{1}, e_{3}\right\} \\ \left\{e_{2}, e_{3}\right\} & 1 & 17 & \left\{e_{2}, e_{3}\right\} \\ \left\{2, e_{2}\right\} & 1 & 1 & \left\{e_{2}, e_{3}\right\} \\ \left\{2, e_{3}\right\} & 1 & 1 & \left\{e_{2}, e_{3}\right\} \\ \left\{6, e_{1}\right\} & 3 & 3 & \left\{6, e_{1}\right\} \\ \left\{6, e_{2}\right\} & 3 & 3 & \left\{6, e_{2}\right\} \\ \left\{4, e_{3}\right\} & 3 & 1 & \left\{6, e_{3}\right\} \\ \left\{8, e_{3}\right\} & 3 & 1 & \left\{6, e_{3}\right\} \\ \left\{5, e_{3}\right\} & 3 & 2 & \left\{6, e_{3}\right\} \\ \left\{7, e_{3}\right\} & 3 & 2 & \left\{6, e_{3}\right\} \\ \{4,5\} & 3 & 1 & \left\{6, e_{3}\right\} \\ \{5,6\} & 9 & 1 & \left\{6, e_{3}\right\} \\ \{6,7\} & 9 & 1 & \left\{6, e_{3}\right\} \\ \{7,8\} & 3 & 1 & \left\{6, e_{3}\right\}\end{array}$

There is considerable overlap among these great circles. For instance, the points 4 and 8 coincide, as do the points 5 and 7 , and they all lie on the great circle between $e_{3}$ and 6 . Likewise, the point 2 lies on the great circle between $e_{2}$ and $e_{3}$. Thus the embedded graph $\mathcal{T}(I) \subset \mathbb{S}^{2}$ is the complete graph $K_{4}$, and, by adding up the contributions of each index times mixed volume, we get the corresponding weights:

$\begin{array}{ccccccc}\text { edge } \Gamma & \left\{e_{1}, e_{2}\right\} & \left\{e_{1}, e_{3}\right\} & \left\{e_{2}, e_{3}\right\} & \left\{6, e_{1}\right\} & \left\{6, e_{2}\right\} & \left\{6, e_{3}\right\} \\ \text { weight } M_{\Gamma} & 9 & 18 & 18 & 9 & 9 & 18\end{array}$

From this table we can now determine the Newton polytope $Q$ of the implicit equation $g\left(x_{1}, x_{2}, x_{3}\right)$. The general method for this is explained in the next section. In this example we find that the proposed Newton polytope is the tetrahedron

$$
Q=\left\{\left(u_{1}, u_{2}, u_{3}\right) \in \mathbb{R}^{3}: u_{1}, u_{2}, u_{3} \geq 0 \text { and } u_{1}+u_{2}+2 \cdot u_{3} \leq 18\right\} .
$$

The number of lattice points in $Q$ equals 715 . What we can conclude at this point is that $Q$ would be the Newton polytope of the implicit equation $g$ if $f_{1}, f_{2}, f_{3}$ were replaced by polynomials with the same support but with generic coefficients.

However, the coefficients of the specific polynomials $f_{1}, f_{2}, f_{3}$ we took from EK, Example 3.4] are not generic. For instance, the equations $f_{2}(s, t)=f_{3}(s, t)=0$ have only 15 solutions $(s, t) \in\left(\mathbb{C}^{*}\right)^{2}$, which is less than the number 17 predicted by the mixed volume. Yet, it turns out that $Q$ is the correct Newton polytope and all 715 possible monomials appear with non-zero coefficients in the implicit equation:

$$
\begin{aligned}
& g\left(x_{1}, x_{2}, x_{3}\right)=387420489 x_{1}^{18}+387420489 x_{2}^{18}-18014398509481984 x_{3}^{9}+\cdots \cdots \\
& \quad-12777985432959891776936639829 x_{2}^{2}+\cdots-3707912273492242256259566313 .
\end{aligned}
$$

We included the $x_{2}^{2}$-term because it has the coefficient of largest absolute value. 


\section{From tropical VAriety to NeWton polytope to implicit equation}

In the previous sections we constructed the tropical variety $\mathcal{T}(I)$ along with the multiplicity $M_{\Gamma}$ for each maximal cone $\Gamma$. Assuming $I=\langle g\rangle$ to be a principal ideal, we now show how this information reveals the Newton polytope $Q$ of $g$. The polynomial $g$ is then recovered from $Q$ and the $f_{i}$ 's using numerical linear algebra. If $V(I)$ is not a hypersurface then the role of $Q$ will be played by the Chow polytope.

5.1. Generic hypersurfaces. The hypersurface case, when $n=d+1$, has recieved the most attention in the computer algebra literature [CGKW, EK SY]. Suppose we are given $d+1$ Laurent polynomials $f_{0}, f_{1}, \ldots, f_{d}$ whose coefficients are generic relative to their supports $A_{0}, A_{1}, \ldots, A_{d} \subset \mathbb{Z}^{d}$. Their Newton polytopes are denoted by $P_{0}, P_{1}, \ldots, P_{d}$. Note that we shifted indices by one. The prime ideal of algebraic relations among the $f_{i}$ is denoted, as before, by $I \subset \mathbb{C}\left[x_{0}, x_{1}, \ldots, x_{d}\right]$. There is a simple combinatorial criterion for when the ideal $I$ is principal, i.e., when the variety $V(I)$ parametrized by $\left(f_{0}, f_{1}, \ldots, f_{d}\right)$ is actually a hypersurface in $\mathbb{C}^{d+1}$.

Proposition 5.1. The prime ideal $I$ is principal if and only if there exist points $a_{i} \in A_{i}$ such that the $d \times(d+1)$-matrix $\left(a_{0}, a_{1}, \ldots, a_{d}\right)$ has maximal rank $d$.

Proof. This can be seen by writing down the $d \times(d+1)$-Jacobian matrix $\left(\partial f_{i} / \partial t_{j}\right)$, and using the fact that the coefficients of the $f_{i}$ are generic.

We now assume that the condition in Proposition 5.1 is satisfied, and we let $g=g\left(x_{0}, x_{1}, \ldots, x_{d}\right)$ denote the unique (up to scaling) generator of the ideal $I$. Let $Q \subset \mathbb{R}^{d+1}$ be the Newton polytope of the irreducible polynomial $g$. Then the tropical hypersurface $\mathcal{T}(I)=\mathcal{T}(g)$ is the union of the codimension one cones in the normal fan of $Q$, and the multiplicity of a maximal cone $\Gamma$ in any fan structure on $\mathcal{T}(I)$ equals to lattice length of the edge of $Q$ whose normal cone contains $\Gamma$.

Suppose we constructed the tropical hypersurface $\mathcal{T}(I)$ using Theorem 2.1 and we computed the multiplicities $M_{\Gamma}$ of each cone $\Gamma$ in $\mathcal{T}(I)$ using Theorem 4.1 These data, combined with the requirement that $Q$ lies in the non-negative orthant and intersects each of the $d+1$ coordinate hyperplanes, determine the polytope $Q$ uniquely. Hence we can construct the desired Newton polytope $Q \subset \mathbb{R}^{d+1}$ combinatorially from the given Newton polytopes $P_{0}, P_{1}, \ldots, P_{d} \subset \mathbb{R}^{d}$.

The previous paragraph almost solves the problem stated in $[\mathrm{SY}]$. The only shortcoming is that the description of the set $\mathcal{T}(I)$ given in Theorem 2.1 does not come with a nice fan structure. We saw this in Examples 3.4 and 4.3 In our view, the following theorem provides a better solution, as it does not require the knowledge of any fan structure. All that is needed are the integers $M_{\gamma}=M_{\Gamma}>0$ where $\gamma$ is a smooth point on $\mathcal{T}(I)$ and $\Gamma$ is any sufficiently small relatively open cone in $\mathcal{T}(I)$ which contains $\gamma$. But these numbers $M_{\gamma}$ are computed using the formula in Theorem 4.1 simply by replacing " $(C, J)$ covers $\Gamma$ " with " $(C, J)$ covers $\gamma "$.

The following theorem gives a formula for each coordinate of each vertex of the Newton polytope $Q$. It does not rely on any particular fan structure on $\mathcal{T}(I)$.

Theorem 5.2. For a generic vector $v \in \mathbb{R}^{d+1}$, the $i^{\text {th }}$ coordinate of the vertex face $_{v}(Q)$ equals the number of intersection points, each counted with its intersection multiplicity, of the tropical hypersurface $\mathcal{T}(I)$ with the halfline $v+\mathbb{R}_{\geq 0} e_{i}$. 
This result and its generalization to larger codimension, to be stated in Theorem [5.5 below, is due to Dickenstein, Feichtner and the first author, and it appears in the paper [DFS] on Tropical Discriminants. We consider the conjunction of Theorems 2.14 .1 and 5.2 to be a satisfactory solution to the problem stated in [SY].

What remains is for us to explain the meaning of the term "intersection multiplicity" in the statement of Theorem 5.2 Since $v$ is generic, each intersection of the tropical hypersurface $\mathcal{T}(I)$ with the halfline $v+\mathbb{R}_{\geq 0} e_{i}$ occurs in a smooth point $\gamma$ of $\mathcal{T}(I)$. The intersection multiplicity at $\gamma$ is the product of the multiplicity $M_{\gamma}$ and the index of the finite index sublattice $\mathbb{Z} e_{i}+\left(\mathbb{R} \Gamma \cap \mathbb{Z}^{d+1}\right)$ of $\mathbb{Z}^{d+1}$. In DFS, $\S 2]$, these two factors were called the intrinsic and extrinsic multiplicities of the intersection.

5.2. Computational issues. Theorems 2.1 and 4.1provide an algorithm for computing the tropical variety $\mathcal{T}(I)$ and the multiplicities $M_{\Gamma}$ for each maximal cone $\Gamma$ in a fan structure of $\mathcal{T}(I)$. The dimension of the fan $\mathcal{T}(I)$ coincides with the dimension of the variety $V(I)$ it represents. When $V(I)$ is a hypersurface, characterized combinatorially by Proposition 5.1 we compute a list of all vertices of the Newton polytope $Q$ by means of Theorem [5.2 All of these computations require only polyhedral geometry and linear algebra (over the integers), but they do not require any methods from computational commutative algebra (Gröbner bases, resultants).

We are in the process of developing an implementation of these algorithms in a software package called $\operatorname{Tr} \operatorname{Im}$ (Tropical Implicitization). A very preliminary test implementation already exists. It is a perl script that incorporate the software packages Polymake GJ], BBMinkSum [Hu, Mixed Volume Library [EC, Maple, and Matlab. We use BBMinkSum to compute Minkowski sums of the input Newton polytopes $P_{i}$ and Polymake to find the face structure of $P=P_{1}+\cdots+P_{n}$. Then for each cone $C$ in the normal fan of $P$, we look for the subsets $J \subset\{1, \ldots, n\}$ with $|J|+\operatorname{dim}(C)=d$ such that $\Psi(C)+\mathbb{R}_{\geq 0}^{J}$ is in $\mathcal{T}(I)$. This amounts to doing linear algebra using Matlab. We then use Mixed Volume Library and Maple to compute the scaled mixed volumes (4.4) for pairs $(C, J) \subset \mathcal{T}(I)$.

The input to TrIm is either a list of Laurent polynomials or their Newton polytopes (in Polymake format). The output is a description of $\mathcal{T}(I)$ in the form of a list of pairs $(C, J)$ as described before. Notice that the output is not the same as the output of Gfan BJSST because we do not get a fan structure from the description in Theorem 2.1 Using this output, we can find the vertices of the Newton polytope using Theorem 5.2 or the vertices of the Chow polytope using Theorem 5.5

In the hypersurface case, the exponents of monomials with non-zero coefficients in the implicit equation $g$ all lie in the Newton polytope $Q$. After computing the vertices of $Q$, enumerating the integer points in $Q$ gives us a list of all possible monomials in $g$ with indeterminate coefficients. Finally, we apply numerical linear algebra to compute the indeterminate coefficients of the polynomial $g$.

This is done as follows. Recall that we are given a parametrization $\left(f_{0}, \ldots, f_{d}\right)$ of the hypersurface $V(I)$. We can thus pick any point $\tau \in\left(\mathbb{C}^{*}\right)^{d}$ and substitute its image $\left(x_{0}, \ldots, x_{d}\right)=\left(f_{0}(\tau), \ldots, f_{d}(\tau)\right) \in V(I)$ into the equation $g\left(x_{0}, \ldots, x_{d}\right)=0$. This gives us one linear equation for the coefficients of $g$. We now pick a different point $\tau^{\prime}$ in $\left(\mathbb{C}^{*}\right)^{d}$ to get a second equation, and so on. In this manner, we can generate a system of linear equations whose solution space is one-dimemensional, and is spanned by the vector of coefficients of the desired implicit equation $g$. 
In our first experiments, we found that this linear algebra problem seems to be numerically stable and efficiently solvable in Matlab if we use vectors $\tau$ whose coordinates $\tau_{i}$ are unitary numbers, i.e., complex numbers of modulus 1 . But there are other possible schemes for generating and solving these linear systems. For instance, the authors of [CGKW] advocate the use of integral operators in setting up linear equations for the coefficients on the implicit equation $g$. We believe that implicitization is a fruitful direction of further study in numerical linear algebra.

5.3. Non-generic hypersurfaces. The following proposition ensures that we can apply Tropical Implicization even if the given polynomials $f_{i}(t)$ do not have generic coefficients. If there is a unique irreducible relation among the given $f_{i}$, then our linear algebra method will find that relation, up to a polynomial multiple.

Proposition 5.3. Let $f_{0}, \ldots, f_{d} \in \mathbb{C}\left[t_{1}^{ \pm 1}, \ldots, t_{d}^{ \pm 1}\right]$ be any Laurent polynomials whose ideal of algebraic relations is principal, say $I=\langle g\rangle$, and $P_{i} \subset \mathbb{R}^{d}$ the Newton polytope of $f_{i}$. Then the polytope $Q$, which is constructed combinatorially from $P_{0}, \ldots, P_{d}$ as in Subsection 5.1 contains a translate of the Newton polytope of $g$.

Proof. We introduce a family of Laurent polynomials $f_{0}^{\epsilon}(t), f_{1}^{\epsilon}(t), \ldots, f_{d}^{\epsilon}(t)$ with coefficients in $\mathbb{C}(\epsilon)$ which are generic for their support and which satisfy $\lim _{\epsilon \rightarrow 0} f_{i}^{\epsilon}(t)=$ $f_{i}(t)$ for all $i$. There exists an irreducible polynomial $g^{\epsilon}\left(x_{0}, x_{1}, \ldots, x_{d}\right)$ with coefficients in $\mathbb{C}(\epsilon)$ which vanishes if we replace each $x_{i}$ by $f^{\epsilon}(t)$. Removing common factors of $\epsilon$ from the terms of $g^{\epsilon}$, we may assume that

$$
\hat{g}(x):=\lim _{\epsilon \rightarrow 0} g^{\epsilon}(x)
$$

is not the zero polynomial. The Newton polytope of $\hat{g}$ is contained in $Q$. Now, we take the limit for $\epsilon \rightarrow 0$ in the identify

$$
g^{\epsilon}\left(f_{0}^{\epsilon}(t), f_{1}^{\epsilon}(t), \ldots, f_{d}^{\epsilon}(t)\right) \equiv 0 \quad \text { in } \mathbb{C}(\epsilon)\left[t_{1}^{ \pm 1}, \ldots, t_{d}^{ \pm 1}\right] .
$$

This implies that $\hat{g}(x)$ is an algebraic relation among $f_{0}(t), f_{1}(t), \ldots, f_{d}(t)$, so the irreducible polynomial $g(x)$ is a factor of $\hat{g}(x)$. This means that the Newton polytope of $g$ is a Minkowski summand of the Newton polytope of $\hat{g}$. This implies that a translate of the Newton polytope of $g$ lies in $Q$.

Example 5.4. Let $d=2$ and consider the three homogeneous quadrics:

$$
\begin{aligned}
& f_{0}(s, t)=s^{2}+s t-2 t^{2} \\
& f_{1}(s, t)=s^{2}-2 s t+t^{2} \\
& f_{2}(s, t)=-2 s^{2}+s t+t^{2}
\end{aligned}
$$

Here, $g=x_{0}+x_{1}+x_{2}$, so its Newton polytope is the triangle $\Delta=\operatorname{conv}\left\{e_{0}, e_{1}, e_{2}\right\}$. The Newton polytope of the implicit equation for a parametric surface given by three general quadrics is twice that triangle: $Q=2 \cdot \Delta$. This shows that $\Delta$ is not a subpolytope of $Q$ but a (non-unique) translate of $\Delta$ is a subpolytope of $Q$.

The approach suggested by Proposition 5.3 is to not worry at all whether the given polynomials have generic coefficients or special coefficients. The idea is that this will not be relevant until the very end, when the numerical linear algebra detects that the solution space to the linear system of equations for the coefficients of $g(x)$ is larger than expected. This should work fine when the $f_{i}$ are not too far from the generic case. However, for the kind of special morphisms $f$ which typically arise in algebraic geometry and its applications (for instance, in statistics), this approach 
is likely to fail in practice. In such cases, one needs to be more clever in designing suitable compactifications $Y$ of the very affine variety $Y^{0}$ of Section 2; see [STY].

5.4. Lower-dimensional varieties. We now consider the case when the parametric variety $V(I) \subset \mathbb{C}^{n}$ and its tropicalization $\mathcal{T}(I) \subset \mathbb{R}^{n}$ do not have codimension one. For technical reasons, we here assume that the $f_{i}$ are homogeneous polynomials of the same degree, so that $I$ is a homogeneous prime ideal in $\mathbb{C}\left[x_{1}, \ldots, x_{n}\right]$. Here the role of the Newton polytope $Q$ is played by the Chow polytope Chow $(I)$. This is the lattice polytope in $\mathbb{R}^{n}$ which is defined as follows. For a generic vector $v \in \mathbb{R}^{n}$, the $i^{\text {th }}$ coordinate of the vertex face $_{v}(\operatorname{Chow}(I))$ of Chow $(I)$ is the sum of $\mu_{P}\left(\operatorname{in}_{v}(I)\right)$ where $P$ runs over all monomial primes that contain the variable $x_{i}$ and $\mu_{P}\left(\operatorname{in}_{v}(I)\right)$ denotes the multiplicy of the monomial ideal $\operatorname{in}_{v}(I)$ along $P$. The Chow polytope was introduced and studied in KSZ. If $V(I)$ has codimension one, then Chow $(I)$ is precisely the Newton polytope of the irreducible generator $g$ of $I$.

The following result from [DFS, Theorem 2.2] shows that in the generic case we can construct Chow $(I)$ from the tropical variety $\mathcal{T}(I)$ and its multiplicities $M_{\gamma}$ in the same way as constructing the Newton polytope of the implicit equation of a hypersurface. Let $I$ be any prime ideal of height $c$ in $\mathbb{C}\left[x_{1}, \ldots, x_{n}\right]$ and $v$ a generic vector in $\mathbb{R}^{n}$, so that the initial ideal $\operatorname{in}_{v}(I)$ is generated by monomials.

Theorem 5.5 (DFS). A prime ideal $P=\left\langle x_{i_{1}}, \ldots, x_{i_{c}}\right\rangle$ is a minimal prime of $\operatorname{in}_{v}(I)$ if and only if the tropical variety $\mathcal{T}(I)$ meets the cone $v+\mathbb{R}_{\geq 0}\left\{e_{i_{1}}, \ldots, e_{i_{c}}\right\}$. The number of intersections, each counted with its intersection multiplicity, coincides with the multiplicity $\mu_{P}\left(\operatorname{in}_{v}(I)\right)$ of the monomial ideal $\operatorname{in}_{v}(I)$ along $P$.

Geometrically, the vertices of Chow $(I)$ corresponds to the toric degenerations of the algebraic cycle underlying $I$. All points in Chow $(I)$ have the same coordinate sum, namely, the degree of the projective variety of $I$. Thus we get a combinatorial rule of computing the degree of the image of any projective morphism. Moreover, if $I$ is homogeneous with respect to some multigrading, then Theorem 5.5 gives us a formula also for the multidegree of $I$. The multidegree is a multivariate polynomial which refines the classical notion of degree, and which has received much attention recently in algebraic combinatorics through the work of Knutson and Miller KM].

\section{REFERENCES}

[Ar] F. Ardila, A tropical morphism related to the hyperplane arrangement of the complete bipartite graph, math.C0/0404287

[AK] F. Ardila and C. Klivans, The Bergman complex of a matroid and phylogenetic trees, J. Combin. Theory Ser. B 96 (2006) 38-49.

[Be] D. Bernstein, The number of roots of a system of equations, Functional Analysis and its Applications 9 (1975) 183-185.

[BJSST] T. Bogart, A. Jensen, D. Speyer, B. Sturmfels and R. Thomas, Computing tropical varieties, Journal of Symbolic Computation (special issue MEGA 2005), to appear.

[Br] R. Brualdi: Introduction to matching theory, in N. White (ed.): Combinatorial Geometries, pp. 53-71, Encyclopedia Math. Appl., 29, Cambridge Univ. Press, Cambridge, 1987.

[CGKW] R. Corless, M. Giesbrecht, I. Kotsireas and S. Watt, Numerical implicitization of parametric hypersurfaces with linear algebra, in: Artificial Intelligence and Symbolic Computation, Springer Lecture Notes in Computer Science, 1930 (2000) 174-183.

[DFS] A. Dickenstein, E.-M. Feichtner and B. Sturmfels, Tropical discriminants, math.AG/0510126

[EC] I.Z. Emiris and J. Canny: Efficient incremental algorithms for the sparse resultant and the mixed volume, Journal of Symbolic Computation 20 (1995) 117-150. 
[EK] I.Z. Emiris and I.S. Kotsireas: Implicitization exploiting sparseness, in D. Dutta, M. Smid, R. Janardan (eds): Geometric And Algorithmic Aspects Of Computer-aided Design And Manufacturing, pp. 281-298, DIMACS Series in Discrete Mathematics and Theoretical Computer Science 67, American Mathematical Society, Providence RI, 2005.

[Ew] G. Ewald, Combinatorial Convexity and Algebraic Geometry, Graduate Texts in Mathematics 168, Springer, New York, 1996.

[GJ] E. Gawrilow and M. Joswig: Polymake: a framework for analyzing convex polytopes, in Polytopes - Combinatorics and Computation, eds. G. Kalai and G.M. Ziegler, Birkhäuser, 2000, pp. 43-74.

[HKT] P. Hacking, S. Keel, and J. Tevelev: Treatise on Terada, Naruki, and Sekiguchi models, in preparation

[HS] B. Huber and B. Sturmfels: A polyhedral method for solving sparse polynomial systems, Mathematics of Computation 64 (1995) 1541-1555.

[Hu] P. Huggins. A program for computing Minkowski sums of polytopes.

[KSZ] M. Kapranov, B. Sturmfels and A. Zelevinsky: Chow polytopes and general resultants, Duke Mathematical Journal 67 (1992) 189-218.

[Ka] E. Katz, The tropical degree of cones in the secondary fan, math.AG/0604290

[Kho] A. Khovanskii: Newton polyhedra, a new formula for mixed volume, product of roots of a system of equations, The Arnoldfest (Toronto, ON, 1997), 325-364, Fields Inst. Commun., 24, Amer. Math. Soc., Providence, RI, 1999.

[KM] A. Knutson and E. Miller: Gröbner geometry of Schubert polynomials, Annals of Mathematics 161 (2005) 1245-1318.

[Mi] G. Mikhalkin, Tropical geometry and its applications, Lecture at the International Congress of Mathematicians, ICM 2006, Madrid,math.AG/0601041

[PS] L. Pachter and B. Sturmfels, Tropical geometry of statistical models, Proceedings of the National Academy of Sciences 101 (2004) 16132-16137.

[Po] A. Postnikov, Permutohedra, associahedra, and beyond, math.C0/0507163

[SS] D. Speyer and B. Sturmfels, The tropical Grassmannian, Advances in Geometry 4 (2004) 389-411.

[STY] B. Sturmfels, J. Tevelev and J. Yu, Tropical implicitization, in preparation.

[SY] B. Sturmfels and J.-T. Yu, Minimal polynomials and sparse resultants, in: "ZeroDimensional Schemes", (eds. F. Orecchia and L. Chiantini), Proceedings Ravello (June 1992), De Gruyter, Berlin, 1994, pp. 317-324.

[Te] E. Tevelev, Compactifications of subvarieties of tori, American J. Math., to appear.

Department of Mathematics, University of California, Berkeley, CA 94720-3840

E-mail address: bernd@math.berkeley.edu

Department of Mathematics, University of Massachusetts, Amherst, MA 01003-9305

E-mail address: tevelev@math.utexas.edu

Department of Mathematics, University of California, Berkeley, CA 94720-3840

E-mail address: jyu@math.berkeley.edu 